\title{
A NOTE ON COVERINGS OF POSETS, A-SPACES AND POLYHEDRA
}

\author{
JONATHAN ARIEL BARMAK AND ELIAS GABRIEL MINIAN
}

\begin{abstract}
We show that there exists a correspondence between the equivalence classes of coverings of a polyhedron and the equivalence classes of coverings of its poset of simplices. The same is true for a poset and its order complex. The coverings of a poset can be understood in two equivalent ways, as categorical coverings, when the poset is viewed as a category, or as topological coverings, when it is viewed as an $A$-space. This implies that the theory of coverings of polyhedra can be handled completely in the combinatorial setting.
\end{abstract}

\section{Introduction}

An $A$-space (or Alexandroff space) is a topological space in which arbitrary intersections of open sets are open. There is a one-to-one correspondence between $A$-spaces and preorders (sets with a reflexive and transitive relation) which was first studied by Alexandroff [1]. In the last fifty years, $A$-spaces have shown to be a useful combinatorial and topological tool in Homotopy Theory (see $[2,3,4,7,8,12]$ ). Concretely, McCord defined in [11] a functor $\mathcal{K}$ which asociates a polyhedron to each $A$-space $X$ and he proved that there is a weak homotopy equivalence $\mu: \mathcal{K}(X) \rightarrow X$. In particular, both spaces have the same homotopy and homology groups. Conversely, for each polyhedron $K$ there exists an $A$-space $\mathcal{X}(K)$ and a weak homotopy equivalence $K \rightarrow \mathcal{X}(K)$. The modest purpose of this note is to show that for every $A$-space $X$, there is a correspondence between the equivalence classes of covering maps of $X$ and of $\mathcal{K}(X)$ and that for every polyhedron $K$, $\mathcal{X}$ establishes a correspondence between classes of coverings of $K$ and of $\mathcal{X}(K)$. Hence, the theory of coverings of polyhedra can be studied in the setting of $A$-spaces. We will first show that the topological notion of covering of an $A$-space $X$ coincides with the categorical notion of covering of the preorder associated to $X$, considered as a category with at most one morphism between any two objects.

\section{Coverings of $A$-spaces and posets}

We recall very briefly the correspondence between $A$-spaces and preorders and the relationship with polyhedra. For more details we refer the reader to $[2,11]$.

Given an $A$-space $X$, for each point $x$ in $X$ let $U_{x}$ be the intersection of all the open sets containing $x$. This is the smallest open set which contains $x$. The preorder associated to the $A$-space $X$ has the same underlying set and the relation is given by $x \leq y$ if $x \in U_{y}$. Conversely, given a preorder $\leq$ on a set $X$, the topology corresponding to this relation is the one generated by the basis given by the sets $U_{x}=\{y \in X \mid y \leq x\}$, for every $x \in X$.

2010 Mathematics Subject Classification. 57M10, 06A07, 55U10, $18 \mathrm{~B} 35$.

Key words and phrases. Covering maps, simplicial complexes, posets, $A$-spaces.

Researchers of CONICET. Partially supported by grants ANPCyT PICT-2011-0812, CONICET PIP 112-201101-00746 and UBACyT 20020130100369. 
These assignments establish a correspondence between $A$-spaces and preorders. It is easy to prove that a function between $A$-spaces is continuous if and only if it is order-preserving. Any $A$-space is locally contractible since the sets $U_{x}$ are contractible (see [11, Lemma 6$]$ ). In particular, any $A$-space has a universal cover.

If $X$ is an $A$-space, the closed sets of $X$ form another topology on the underlying set of $X$, called the opposite topology. The preorder associated to this topology is the opposite order of $X$. This space is denoted by $X^{o p}$. Note that a map $f: X \rightarrow Y$ between $A$-spaces is continuous if and only if the induced map $f^{o p}: X^{o p} \rightarrow Y^{o p}$, which coincides with $f$ in the underlying sets, is continuous. If $X$ is an $A$-space, the closure of a point $x$ in $X$ is denoted by $F_{x}$. Note that $F_{x}^{X}=\{y \in X, x \leq y\}=\left(U_{x}^{X^{o p}}\right)^{o p}$. The notations $F_{x}^{X}$ and $U_{x}^{X}$ will be used when we need to emphasize the space $X$ where these subsets are considered.

Recall that a topological space $X$ is said to be $T_{0}$ if for any two points $x, y \in X$ there is an open set which contains one and only one of them. If an $A$-space is $T_{1}$, it is discrete, so $T_{0}$ is the unique separation axiom that we will consider. It is easy to prove an $A$-space is $T_{0}$ if and only if the corresponding preorder is a poset, that is, the relation is also antisymmetric.

Proposition 1. Let $p: E \rightarrow B$ be a (topological) covering map. If $B$ is an A-space, then so is $E$.

Proof. Let $\left\{U_{\alpha}\right\}$ be an arbitrary family of open sets of $E$. If $\cap U_{\alpha}$ is empty, there is nothing to prove. Suppose $\bigcap U_{\alpha}$ is non-empty and let $e \in \bigcap U_{\alpha}$. Let $U$ be an open neighborhood of $e$ and $V=p(U)$ an open neighborhood of $p(e)$ such that $\left.p\right|_{U}: U \rightarrow V$ is a homeomorphism. Then $p\left(U \cap U_{\alpha}\right)$ is an open subset of $B$ for every $\alpha$, and since $B$ is an $A$-space, $\bigcap p\left(U \cap U_{\alpha}\right)=p\left(U \cap \cap U_{\alpha}\right)$ is open. Thus, $U \cap \bigcap U_{\alpha}$ is an open neighborhood of $e$ contained in $\bigcap U_{\alpha}$. This shows that $\bigcap U_{\alpha}$ is open.

With the same proof one can see that the last proposition remains valid when $p$ is just a local homeomorphism. Moreover if $f: X \rightarrow Y$ is a surjective local homeomorphism and $X$ is an $A$-space, then so is $Y$.

Remark 2. Let $p: E \rightarrow B$ be a covering of a topological space $B$. If $B$ is $T_{0}$, so is $E$. It is clear that two points of $E$ in different fibers can be separated since $B$ is $T_{0}$. If two points are in the same fiber, they can also be separated since $p$ is a covering.

The following elementary result is well-known.

Remark 3. Let $p: E \rightarrow B$ be a covering and suppose $A$ is a connected and locally connected subspace of $B$. If $C$ is a connected component of $p^{-1}(A)$, then $\left.p\right|_{C}: C \rightarrow A$ is a covering.

Proposition 4. Let $B$ be an $A$-space and let $p: E \rightarrow B$ be a covering. Then $p^{o p}: E^{o p} \rightarrow$ $B^{o p}$ is also a covering.

Proof. It follows from the fact that the closure $F_{b}^{B}$ of every $b \in B$ is simply-connected and the previous remark (see also Theorem 13).

All the categories that we work with will be assumed to be small. Note that a preorder $(X, \leq)$ can be regarded as a category $\mathcal{C}$ whose set of objects $\operatorname{Obj}(\mathcal{C})$ is $X$ and with a unique morphism from an object $x$ to an object $y$ if $x \leq y$. Conversely, any category $\mathcal{C}$ which satisfies that for any two objects $x, y$ there is at most one morphism $\alpha \in \operatorname{Mor}(\mathcal{C})$ whose source $\mathrm{s}(\alpha)$ is $x$ and whose target $\mathrm{t}(\alpha)$ is $y$, arises from a preorder in this way. A preorder, 
viewed as a category, is a poset if it has no isomorphisms other than the identities. Note also that order-preserving maps between two preorders correspond to functors between the categories.

We investigate now the relationship between topological coverings of $A$-spaces and coverings of categories. The definition of coverings for categories that we use extends the definition of coverings for groupoids $[6,9,10]$ and it is analogous to the definition of coverings for $k$-categories given in [5].

Given a category $\mathcal{C}$ and $x \in \operatorname{Obj}(\mathcal{C})$, we define the sets $\mathcal{U}_{x}=\{\alpha \in \operatorname{Mor}(\mathcal{C}) \mid \mathrm{t}(\alpha)=x\}$ and $\mathcal{F}_{x}=\{\alpha \in \operatorname{Mor}(\mathcal{C}) \mid \mathrm{s}(\alpha)=x\}$. Recall that $\mathrm{s}(\alpha)$ and $\mathrm{t}(\alpha)$ denote respectively the source and the target of $\alpha$. Note that any functor $F: \mathcal{C} \rightarrow \mathcal{D}$ induces (set theoretic) functions $F_{\mathcal{U}_{x}}: \mathcal{U}_{x} \rightarrow \mathcal{U}_{F(x)}$ and $F_{\mathcal{F}_{x}}: \mathcal{F}_{x} \rightarrow \mathcal{F}_{F(x)}$.

Definition 5. A functor $F: \mathcal{C} \rightarrow \mathcal{D}$ is a covering if it is surjective on objects and $F_{\mathcal{U}_{x}}$ and $F_{\mathcal{F}_{x}}$ are bijections for each $x \in \operatorname{Obj}(\mathcal{C})$.

Proposition 6. Let $\mathcal{D}$ be a category which is a preorder and let $F: \mathcal{C} \rightarrow \mathcal{D}$ be a covering (in the categorical sense). Then $\mathcal{C}$ is also a preorder.

Proof. If $\alpha$ and $\beta$ are morphisms from an object $x$ to an object $y$ in $\mathcal{C}$, then $F(\alpha), F(\beta) \in$ $\operatorname{Mor}(F(x), F(y))$. Since $\mathcal{D}$ is a preorder, $F(\alpha)=F(\beta)$ and since $F_{\mathcal{F}_{x}}$ is a bijection, $\alpha=\beta$.

Example 7. The following functor $F$, which maps $x_{i}$ to $x, y_{i}$ to $y, \alpha_{i}$ to $\alpha$ and $\beta_{i}$ to $\beta$, is a covering from a preorder onto a category which is not a preorder.

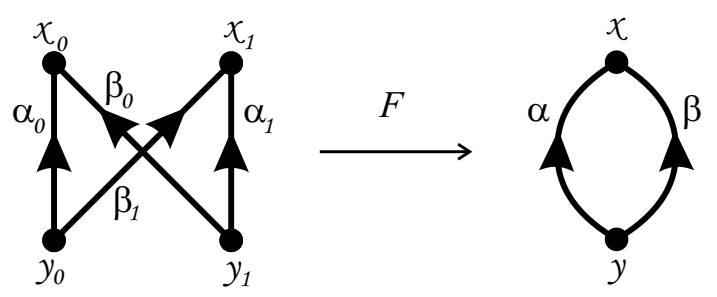

Figure 1. A covering from a poset to a category which is not a preorder.

Lemma 8. When $\mathcal{C}$ and $\mathcal{D}$ are preorders, an order-preserving map $f$ between $\mathcal{C}$ and $\mathcal{D}$ is a covering in the categorical sense if and only if it is surjective and for each $x \in \operatorname{Obj}(\mathcal{C})$, both $\left.f\right|_{U_{x}}: U_{x} \rightarrow U_{f(x)}$ and $\left.f\right|_{F_{x}}: F_{x} \rightarrow F_{f(x)}$ are bijections.

Proof. Since $\mathcal{C}$ and $\mathcal{D}$ are preorders, for each $x \in \operatorname{Obj}(\mathcal{C})$, the source maps s $: \mathcal{U}_{x} \rightarrow U_{x}$ and s : $\mathcal{U}_{f(x)} \rightarrow U_{f(x)}$ are bijections. Moreover, $\left.f\right|_{U_{x}} \mathrm{~s}=\mathrm{s} f_{\mathcal{U}_{x}}$. Then $f_{\mathcal{U}_{x}}: \mathcal{U}_{x} \rightarrow \mathcal{U}_{f(x)}$ is a bijection if and only if $\left.f\right|_{U_{x}}: U_{x} \rightarrow U_{f(x)}$ is a bijection. A similar argument shows that $f_{\mathcal{F}_{x}}$ is a bijection if and only if $\left.f\right|_{F_{x}}$ is.

Proposition 9. Let $f: X \rightarrow Y$ be an order-preserving map between preorders. Then, $f$ is a covering of A-spaces in the topological sense if and only if it is a covering in the categorical sense.

Proof. Assume it is a topological covering and let $x \in X$. Then $U_{f(x)}$ is evenly covered, i.e. $f^{-1}\left(U_{f(x)}\right)$ is a disjoint union $\amalg V_{i}$ of open subsets of $X$ which are mapped homeomorphically to $U_{f(x)}$ by $f$. Suppose $x \in V_{i}$. Then $U_{x} \subseteq V_{i}$ and, since $f\left(U_{x}\right)$ is an open 
set which contains $f(x), f\left(U_{x}\right)=U_{f(x)}$ and $\left.f\right|_{U_{x}}: U_{x} \rightarrow U_{f(x)}$ is a homeomorphism. By Proposition 4, the same argument shows that $\left.f\right|_{F_{x}}: F_{x} \rightarrow F_{f(x)}$ is a homeomorphism. By Lemma $8, f$ is a covering in the categorical sense.

Conversely, suppose $f$ is a covering in the categorical sense. By Lemma $8,\left.f\right|_{U_{x}}: U_{x} \rightarrow$ $U_{f(x)}$ and $\left.f\right|_{F_{x}}: F_{x} \rightarrow F_{f(x)}$ are bijections for every $x \in X$. Let $y \in Y$. We will prove that $f^{-1}\left(U_{y}\right)=\coprod_{x \in f^{-1}(y)} U_{x}$ and that the restrictions $\left.f\right|_{U_{x}}: U_{x} \rightarrow U_{y}$ are homeomorphisms for every $x \in f^{-1}(y)$. It is clear that $\bigcup_{x \in f^{-1}(y)} U_{x} \subseteq f^{-1}\left(U_{y}\right)$ because $f$ is order-preserving. Let $x^{\prime} \in X$ be such that $f\left(x^{\prime}\right) \leq y$. Since $\left.f\right|_{F_{x^{\prime}}}: F_{x^{\prime}} \rightarrow F_{f\left(x^{\prime}\right)}$ is surjective, there exists $x \in F_{x^{\prime}}$ such that $f(x)=y$. Therefore, $x^{\prime} \in U_{x}$ with $x \in f^{-1}(y)$. This proves that $f^{-1}\left(U_{y}\right) \subseteq \bigcup_{x \in f^{-1}(y)} U_{x}$. We show that the union is disjoint. Suppose $x^{\prime} \in U_{x_{1}} \cap U_{x_{2}}$ for $x_{1}, x_{2} \in f^{-1}(y)$. Since $\left.f\right|_{F_{x^{\prime}}}$ is injective, $x_{1}=x_{2}$. In order to show that $\left.f\right|_{U_{x}}: U_{x} \rightarrow U_{f(x)}$ is a homeomorphism, it only remains to see that it is open. This is clear since for any $x^{\prime} \leq x, f\left(U_{x^{\prime}}^{U_{x}}\right)=f\left(U_{x^{\prime}}^{X}\right)=U_{f\left(x^{\prime}\right)}^{Y}$ is open.

Remark 10. From the proof of Proposition 9 we deduce that if $p: E \rightarrow B$ is a covering between $A$-spaces, then for every $b \in B, U_{b}$ is evenly covered by $p^{-1}\left(U_{b}\right)=\coprod_{e \in p^{-1}(b)} U_{e}$. Similarly $F_{b}$ is evenly covered by $p^{-1}\left(F_{b}\right)=\coprod_{e \in p^{-1}(b)} F_{e}$.

Given an $A$-space $X$, there is a quotient $X_{0}$ of $X$, which is also a strong deformation retract of $X$, and consists of one representative in each equivalence class of the relation defined by $x \sim y$ if $x \leq y$ and $y \leq x$. The homotopy that leaves every point of $X$ fixed for $t<1$ and maps $x \in X$ to the representative of its class for $t=1$ is continuous (see [11, Lemma 9]). By definition, $X_{0}$ is a $T_{0}$-space. There is a correspondence between coverings of $X$ and coverings of $X_{0}$ which associates to each covering $p: Y \rightarrow X$, the covering $\left.p\right|_{p^{-1}\left(X_{0}\right)}: p^{-1}\left(X_{0}\right) \rightarrow X_{0}$. To obtain the covering of $X$ corresponding to a covering $p: Y \rightarrow X_{0}$ we only need to add for every point $y$ in the fiber $p^{-1}(x)$ one point $y^{\prime}$ equivalent to $y$ for each point $x^{\prime} \sim x$ in $X$. Therefore, in order to investigate coverings of $A$-spaces it suffices to study coverings of $T_{0}$ - $A$-spaces (i.e. posets).

Corollary 11. Let $f: X \rightarrow Y$ be a continuous map between $T_{0}$-A-spaces. Then $f$ is a covering if and only if it is surjective and the preimage $f^{-1}(c)$ of every finite chain $c$ of $Y$ is a disjoint union of chains of $X$ (with the subspace topology) which are mapped homeomorphically to $c$ by $f$.

Proof. If $f$ is a covering and $c$ is a chain of $Y$ with maximum $y$, then $f^{-1}\left(U_{y}\right)=\coprod_{x \in f^{-1}(y)} U_{x}$ and $f^{-1}(c)=\coprod_{x \in f^{-1}(y)}\left(\left.f\right|_{U_{x}}\right)^{-1}(c)$. Conversely, assume $f$ is surjective and that the preimage of any finite chain $c$ is a disjoint union of chains mapped homeomorphically to $c$. In particular, if $x \in X$, for every $y \in U_{f(x)}$ we have that $f^{-1}(\{y, f(x)\})$ is a disjoint union of chains, of length 1 if $y \neq f(x)$ and of length 0 if $y=f(x)$. Since $x$ is the maximum of one of these chains, then there is one and only one element of $U_{x}$ which is mapped by $f$ to $y$. Thus, $\left.f\right|_{U_{x}}: U_{x} \rightarrow U_{f(x)}$ is a bijection. The same is true for $\left.f\right|_{F_{x}}: F_{x} \rightarrow F_{f(x)}$ and therefore, $f$ is a covering by Proposition 9 . 


\section{Relationship with simplicial coverings}

The order complex functor $\mathcal{K}$ is defined as follows. Given a $T_{0}$ - $A$-space $X, \mathcal{K}(X)$ is the simplicial complex of non-empty finite chains of $X$. Given a continuous map $f: X \rightarrow Y$ between $T_{0}$ - $A$-spaces, $\mathcal{K}(f): \mathcal{K}(X) \rightarrow \mathcal{K}(Y)$ is the simplicial map which maps a vertex $x \in X$ to $f(x) \in Y$. In the other direction, if $K$ is a simplicial complex, its face poset $\mathcal{X}(K)$ is the poset of simplices of $K$ ordered by inclusion. If $\varphi: K \rightarrow L$ is a simplicial map between simplicial complexes, $\mathcal{X}(\varphi): \mathcal{X}(K) \rightarrow \mathcal{X}(L)$ is the continuous map which maps a simplex $\sigma \in K$ to $\varphi(\sigma) \in L$. Before studying the relationship between coverings of polyhedra and of $A$-spaces we recall a characterization of the first. A simplicial map $\varphi: L \rightarrow K$ is a covering if and only if the preimage of every simplex $\sigma \in K$ is a disjoint union of simplices which are mapped isomorphically to $\sigma$. Although this simple fact is known, we could not find a proof in the literature, so we include one here for the sake of completeness.

Let $K$ be a simplicial complex. Every (topological) covering of $K, p: E \rightarrow K$, is equivalent to a covering $\varphi: L \rightarrow K$ where $L$ is a simplicial complex and $\varphi$ is a simplicial map ([13, Theorem 3.8.3]). Recall that two coverings $p: E \rightarrow B$ and $p^{\prime}: E^{\prime} \rightarrow B$ of the same base space $B$ are equivalent if there is a homeomorphism $h: E \rightarrow E^{\prime}$ such that $p^{\prime} h=p$.

Given a simplicial map $\varphi: L \rightarrow K$, and a simplex $\sigma \in K$, we denote by $\varphi^{-1}(\sigma)$ the subcomplex of $L$ whose simplices are those simplices $\tau \in L$ such that $\varphi(\tau) \subseteq \sigma$. Note that the geometric realization of $\varphi^{-1}(\sigma)$ is the preimage of the closed simplex $\bar{\sigma}$ under the geometric realization of $\varphi$.

Proposition 12. Let $\varphi: L \rightarrow K$ be a simplicial map. Then $\varphi$ is a covering if and only if it is surjective on vertices and for every simplex $\sigma$ of $K, \varphi^{-1}(\sigma)$ is a disjoint union of simplices of $L$, each of which is mapped isomorphically to $\sigma$ by $\varphi$.

Proof. If $\varphi: L \rightarrow K$ is a covering and $\sigma \in K$, then by Remark $3 \varphi^{-1}(\sigma)$ is a subcomplex of $L$ which is a disjoint union of subcomplexes $\tau_{i}$ mapped homeomorphically to $\sigma$ by $\varphi$. Then they are mapped isomorphically, and the subcomplexes $\tau_{i}$ are simplices.

Conversely, assume that $\varphi$ is surjective on vertices and that for each simplex $\sigma \in K$, $\varphi^{-1}(\sigma)=\coprod \tau_{i}$ and $\left.\varphi\right|_{\tau_{i}}: \tau_{i} \rightarrow \sigma$ are isomorphisms. Clearly $\varphi$ is surjective. We will show that there is an open cover of $K$ by sets which are evenly covered. Concretely, we will see that for each $v \in K$, the preimage of the open star of a vertex $v \in K$ is

$$
\varphi^{-1}(\stackrel{\circ}{\operatorname{st}}(v))=\coprod_{\varphi(w)=v} \stackrel{\circ}{\operatorname{st}(w)}
$$

and that the restrictions $\stackrel{\circ}{\operatorname{st}}(w) \rightarrow \stackrel{\circ}{\operatorname{st}(v)}$ of $\varphi$ are homeomorphisms. Recall that the open star of the vertex $v$ is the union of the open simplices containing $v$. If $w, w^{\prime}$ are in the fiber of $v$ and their open stars intersect, then $\left\{w, w^{\prime}\right\}$ is a simplex of $\varphi^{-1}(v)$. Since this is by assumption a union of 0 -simplices, $w=w^{\prime}$. Therefore the union is disjoint. The fact that the equality in (1) holds is a general fact for any simplicial map. Let $w \in \varphi^{-1}(v)$. In order to show that $\operatorname{st}(w) \rightarrow \stackrel{\circ}{\operatorname{st}(v)}$ is a homeomorphism, we will prove that the restriction of $\varphi$ to the closed stars $\operatorname{st}(w) \rightarrow \operatorname{st}(v)$ is an isomorphism. Let $v^{\prime}$ be a vertex in $\operatorname{st}(v)$, that is to say $\left\{v, v^{\prime}\right\}$ is a simplex of $K$. If $v \neq v^{\prime}, \varphi^{-1}\left(\left\{v, v^{\prime}\right\}\right)$ is a disjoint union of 1-simplices. Therefore, there exists a unique vertex $w^{\prime} \in \operatorname{st}(w)$ such that $\varphi\left(w^{\prime}\right)=v^{\prime}$. Define $\psi: \operatorname{st}(v) \rightarrow \operatorname{st}(w)$ by $\psi\left(v^{\prime}\right)=w^{\prime}$ and $\psi(v)=w$. This map is simplicial since for 
a $k$-dimensional simplex $\sigma=\left\{v_{0}, v_{1}, \ldots, v_{k}\right\} \in \operatorname{st}(v)$ containing $v, \varphi^{-1}(\sigma)$ is a disjoint union of $k$-simplices and then there exists a unique $k$-simplex $\tau \in L$ containing $w$ and which is mapped isomorphically to $\sigma$ by $\varphi$. Thus, $\psi(\sigma)=\tau$. Clearly $\psi$ is the inverse of $\varphi$, so $\left.\varphi\right|_{\operatorname{st}(w)}: \operatorname{st}(w) \rightarrow \operatorname{st}(v)$ is an isomorphism. This homeomorphism restricts to a homeomorphism $\stackrel{\circ}{\operatorname{st}}(w) \rightarrow \varphi(\stackrel{\circ}{\operatorname{st}(w)})=\stackrel{\circ}{\operatorname{st}}(v)$.

Theorem 13. Let $B$ be a $T_{0}$-A-space. If $p: E \rightarrow B$ is a covering, then $\mathcal{K}(p): \mathcal{K}(E) \rightarrow$ $\mathcal{K}(B)$ is a covering. Moreover, the functor $\mathcal{K}$ establishes a one-to-one correspondence between equivalence classes of coverings of $B$ and equivalence classes of coverings of $\mathcal{K}(B)$.

Proof. Let $p: E \rightarrow B$ be a covering. The map $\mathcal{K}(p)$ is surjective on vertices. Moreover, by Corollary 11 the preimage of every simplex $\sigma \in \mathcal{K}(B)$ is a disjoint union of simplices mapped isomorphically to $\sigma$. Therefore $\mathcal{K}(p)$ is a covering.

Equivalent coverings of $B$ are mapped to equivalent coverings of $\mathcal{K}(B)$. Suppose now that $p_{1}: E_{1} \rightarrow B$ and $p_{2}: E_{2} \rightarrow B$ are coverings of $B$ such that there is a homeomorphism $h: \mathcal{K}\left(E_{1}\right) \rightarrow \mathcal{K}\left(E_{2}\right)$ with $\mathcal{K}\left(p_{2}\right) h=\mathcal{K}\left(p_{1}\right)$. It is easy to see that $h$ is a simplicial map, so it induces a function $f: E_{1} \rightarrow E_{2}$. We prove that $f$ is continuous. If $e<e^{\prime}$ in $E_{1}$, $\left\{e, e^{\prime}\right\}$ is a simplex of $\mathcal{K}\left(E_{1}\right)$ and $h\left(\left\{e, e^{\prime}\right\}\right)=\left\{f(e), f\left(e^{\prime}\right)\right\}$ is then a simplex of $\mathcal{K}\left(E_{2}\right)$. If $f(e) \geq f\left(e^{\prime}\right)$, then $p_{1}(e)=p_{2} f(e) \geq p_{2} f\left(e^{\prime}\right)=p_{1}\left(e^{\prime}\right)$ which is a contradiction since $p_{1}$ is a covering. Thus, $f(e) \leq f\left(e^{\prime}\right)$. Symmetrically, the inverse of $h$ induces an order-preserving map $g: E_{2} \rightarrow E_{1}$ and this is the inverse of $f$. This shows that the mapping from classes of coverings of $B$ to classes of coverings of $\mathcal{K}(B)$ is injective. To check surjectivity, consider a covering of $\mathcal{K}(B)$. It is equivalent to a simplicial covering $\varphi: K \rightarrow \mathcal{K}(B)$. Define an order in the vertex set $E$ of $K$ by $v \leq v^{\prime}$ if $\left\{v, v^{\prime}\right\}$ is a simplex of $K$ and $\varphi(v) \leq \varphi\left(v^{\prime}\right)$. This relation in transitive since if $v \leq v^{\prime} \leq v^{\prime \prime}$, then $v, v^{\prime}$ and $v^{\prime \prime}$ lie in one simplex of $\varphi^{-1}\left(\left\{\varphi(v), \varphi\left(v^{\prime}\right), \varphi\left(v^{\prime \prime}\right)\right\}\right)$. It is not hard to see that $\mathcal{K}(E)=K$. Moreover the map $p: E \rightarrow B$ induced by the map $\varphi$ in the vertices, is a covering by Corollary 11 and Proposition 12 .

Note that, the fact that $\mathcal{K}$ preserves coverings is a particular case of a more general result. It is not hard to see that the nerve functor from the category of small categories to the category of simplicial sets and the geometric realization functor from simplicial sets to spaces preserve coverings (see [9] for more details on the geometric realization functor and coverings of simplicial sets).

Theorem 14. Let $K$ be a simplicial complex. If $\varphi: L \rightarrow K$ is a simplicial covering, then $\mathcal{X}(\varphi): \mathcal{X}(L) \rightarrow \mathcal{X}(K)$ is a covering. Moreover, the functor $\mathcal{X}$ establishes a one-to-one correspondence between equivalence classes of coverings of $K$ and equivalence classes of coverings of $\mathcal{X}(K)$.

Proof. Suppose $\varphi: L \rightarrow K$ is a covering. Clearly $\mathcal{X}(\varphi)$ is surjective. If $\sigma$ is a simplex of $K, \varphi^{-1}(\sigma)=\coprod \tau_{i}$ and $\left.\varphi\right|_{\tau_{i}}: \tau_{i} \rightarrow \sigma$ is an isomorphism. Then $\mathcal{X}(\varphi)^{-1}\left(U_{\sigma}\right)=\coprod U_{\tau_{i}}$, and $\left.\mathcal{X}(\varphi)\right|_{U_{\tau_{i}}}: U_{\tau_{i}} \rightarrow U_{\sigma}$ is a homeomorphism for every $i$. Thus $\mathcal{X}(\varphi)$ is a covering.

Equivalent coverings of $K$ are mapped to equivalent coverings of $\mathcal{X}(K)$. If $h: \mathcal{X}\left(L_{1}\right) \rightarrow$ $\mathcal{X}\left(L_{2}\right)$ is a homeomorphism such that $\mathcal{X}\left(\varphi_{2}\right) h=\mathcal{X}\left(\varphi_{1}\right)$ for some coverings $\varphi_{1}$ and $\varphi_{2}$ of $K$, we show that the latter two are equivalent. Since $\mathcal{X}\left(\varphi_{2}\right) h=\mathcal{X}\left(\varphi_{1}\right)$ where $\mathcal{X}\left(\varphi_{1}\right)$ and $\mathcal{X}\left(\varphi_{2}\right)$ are coverings, $h$ maps minimal elements of $\mathcal{X}\left(L_{1}\right)$ to minimal elements of $\mathcal{X}\left(L_{2}\right)$. Thus, it induces a vertex map $\psi: L_{1} \rightarrow L_{2}$. Moreover, since $h$ is order-preserving, bounded 
sets of minimal elements are mapped to bounded sets. Therefore, $\psi$ is simplicial. The inverse of $h$ induces a simplicial map $L_{2} \rightarrow L_{1}$ which is the inverse of $\psi$, so $\varphi_{1}$ and $\varphi_{2}$ are equivalent.

Suppose now that $p: X \rightarrow \mathcal{X}(K)$ is a covering. Define the complex $L$ whose vertices are the minimal elements of $X$ and whose simplices are the bounded sets of minimal elements (cf. [2, Section 9.2]). Since $p$ is a covering, it maps minimal elements to minimal elements, so it determines a vertex map $\varphi: L \rightarrow K$, which is clearly simplicial since an upper bound of $\left\{x_{0}, x_{1}, \ldots, x_{k}\right\}$ is mapped by $p$ to an upper bound of $\left\{p\left(x_{0}\right), p\left(x_{1}\right), \ldots, p\left(x_{k}\right)\right\}$. We will prove that $\varphi$ is a covering and that $\mathcal{X}(\varphi)$ is equivalent to $p$. Define a map $f: X \rightarrow \mathcal{X}(L)$ which maps an element $x \in X$ to the set of minimal elements of $X$ smaller than $x$. This is an order-preserving map. Moreover, $\mathcal{X}(\varphi) f=p$. If $x \in X$, and $\left\{x_{0}, x_{1}, \ldots, x_{k}\right\}$ is the set of minimal elements below $x$, then $\mathcal{X}(\varphi) f(x)=\left\{p\left(x_{0}\right), p\left(x_{1}\right), \ldots, p\left(x_{k}\right)\right\}$. On the other hand, since $\left.p\right|_{U_{x}}: U_{x} \rightarrow U_{p(x)}$ is a homeomorphism, $\left\{p\left(x_{0}\right), p\left(x_{1}\right), \ldots, p\left(x_{k}\right)\right\}$ is exactly the set of minimal elements of $\mathcal{X}(K)$ smaller that $p(x)$, so $p(x)$ is the simplex $\left\{p\left(x_{0}\right), p\left(x_{1}\right), \ldots, p\left(x_{k}\right)\right\}$. Define now $g: \mathcal{X}(L) \rightarrow X$ as follows. Let $\sigma=\left\{x_{0}, x_{1}, \ldots, x_{k}\right\}$ be a simplex of $L$. Since $\sigma$ has an upper bound $x$ in $X, \tau=\left\{p\left(x_{0}\right), p\left(x_{1}\right), \ldots, p\left(x_{k}\right)\right\}$ is a simplex of $K$. There exists a unique upper bound $x^{\prime}$ of $\left\{x_{0}, x_{1}, \ldots, x_{k}\right\}$ in $X$ such that $p\left(x^{\prime}\right)=\tau$. In fact, the preimage of $\tau$ through $\left.p\right|_{U_{x}}: U_{x} \rightarrow U_{p(x)}$ satisfies that property, and there is at most one since the minimal open sets $U_{x^{\prime}}$ and $U_{x^{\prime \prime}}$ of elements in the same fiber must be disjoint. Define $g\left(\left\{x_{0}, x_{1}, \ldots, x_{k}\right\}\right)=x^{\prime}$. It is easy to see that $g$ is continuous. Furthermore, $g$ is the inverse of $f$. This shows that $\mathcal{X}(\varphi)$ is equivalent to $p$. Finally, since $\mathcal{X}(\varphi)$ is a covering, by Theorem $13 \varphi^{\prime}=\mathcal{K}(\mathcal{X}(\varphi))$ is a covering. The maps $\varphi, \varphi^{\prime}: L=L^{\prime} \rightarrow K=K^{\prime}$ differ only in a homeomorphism. Then $\varphi$ is also a covering.

\section{REFERENCES}

[1] P.S. Alexandroff. Diskrete Räume. Math. Sb. (N.S.) 2 (1937), 501-518.

[2] J.A. Barmak. Algebraic topology of finite topological spaces and applications. Lecture Notes in Mathematics Vol. 2032. Springer (2011) xviii+170 pp.

[3] J.A. Barmak. On Quillen's Theorem A for posets. J. Combin. Theory Ser. A 118(2011), 2445-2453.

[4] J.A. Barmak and E.G. Minian. Simple homotopy types and finite spaces. Adv. Math. 218 (2008), Issue $1,87-104$.

[5] K. Bongartz and P. Gabriel. Covering spaces in representation-theory. Invent. Math. 65 (1981/82), no. 3, 331-378

[6] R. Brown. Topology and groupoids. Booksurge LLC (2006) xxvi+512 pp.

[7] M. Chari. On discrete Morse functions and combinatorial decompositions. Discrete Math. 217 (2000) 101-113.

[8] R. Forman. Morse theory for cell complexes. Adv. Math. 134 (1998) 90-145.

[9] P. Gabriel and M. Zisman. Calculus of fractions and homotopy theory. Ergebnisse der Mathematik und ihrer Grenzgebiete, Band 35 Springer-Verlag (1967). x+168 pp.

[10] J. P. May. A concise course in algebraic topology. Chicago Lectures in Mathematics. University of Chicago Press. (1999). x+243 pp.

[11] M.C. McCord. Singular homology groups and homotopy groups of finite topological spaces. Duke Math. J. 33 (1966), 465-474.

[12] D. Quillen. Homotopy properties of the poset of nontrivial p-subgroups of a group. Adv. Math. 28 (1978) 101-128.

[13] E. Spanier. Algebraic Topology. Springer (1966) ix+528 pp.

Departamento de Matemática-Imas, FCEyn, Universidad de Buenos Aires, Buenos Aires, Argentina

E-mail address: jbarmak@dm.uba.ar

E-mail address: gminian@dm.uba.ar 